\title{
Pensononowoor
}

2017, vol. 77, 19-32

http://dx.doi.org/10.12657/denbio.077.002

\author{
Hédia Hannachi, José Javier Martín Gómez, Ezzeddine Saadaoui, \\ Emilio Cervantes*
}

\section{Stone diversity in wild and cultivated olive trees (Olea europaea L.)}

Received: 30 May 2016; Accepted: 18 November 2016

\begin{abstract}
The olive tree is represented in Tunisia by two varieties: var. sylvestris (wild olive trees) and var. europaea (cultivated olive trees including diverse cultivars). Seed (stone) size and shape analysis may provide the basis for relationships between varieties and cultivars as well as to study the responses to environmental conditions. A semi-automated method of image analysis allows to obtain data related with magnitudes descriptive of stone size and shape and to compare between wild and cultivated olives. Also, the effect of bioclimate on size and shape was analyzed in some cultivars. Stone size and shape presents high variability. In cultivated trees stones are larger. Mean seed image area is 0.38 and $0.75 \mathrm{~cm}^{2}$ for wild and cultivated plants respectively. Roundness and circularity were compared as to their potential to define seed shape. Mean values were higher for circularity, but roundness is more variable reaching higher values in some individuals and varieties. Roundness is more useful to compare seed shape variations. In addition, climate factors affect the stone characteristics in cultivars; those of sub-humid region having larger stones. Phenotypic parameters of stone are discriminating parameters for the analysis of intra-specific, intra-varieties and intra-cultivars variability in Olea europaea.
\end{abstract}

Keywords: Olea europaea, stone, diversity, roundness, circularity, size, Tunisia

Addresses: H. Hannachi, University of Gabès, Faculty of Sciences, URCMEP, Tunisia, e-mail: hannachi hedia@yahoo.fr

J. J. Martín Gómez, IRNASA-CSIC, Cordel de Merinas, 40. 37008, Salamanca, Spain, e-mail: jjavier. martin@irnasa.csic.es

E. Cervantes, IRNASA-CSIC, Cordel de Merinas, 40.37008, Salamanca, Spain, e-mail: ecervant@usal.es E. Saadaoui, University of Carthage, National Institute of Research in Rural Engineering, Waters and Forests (INRGREF), LGVRF, Regional Station of Gabès, Tunisia, e-mail: saad ezz@yahoo.fr

*Corresponding author

\section{Introduction}

Olive (Olea europaea, L.) is one of the most important crops in Mediterranean countries. The olives are used for table consumption as well as an important source of oil. The olive tree has a long tradition of historic and cultural significance (Fig. 1). 

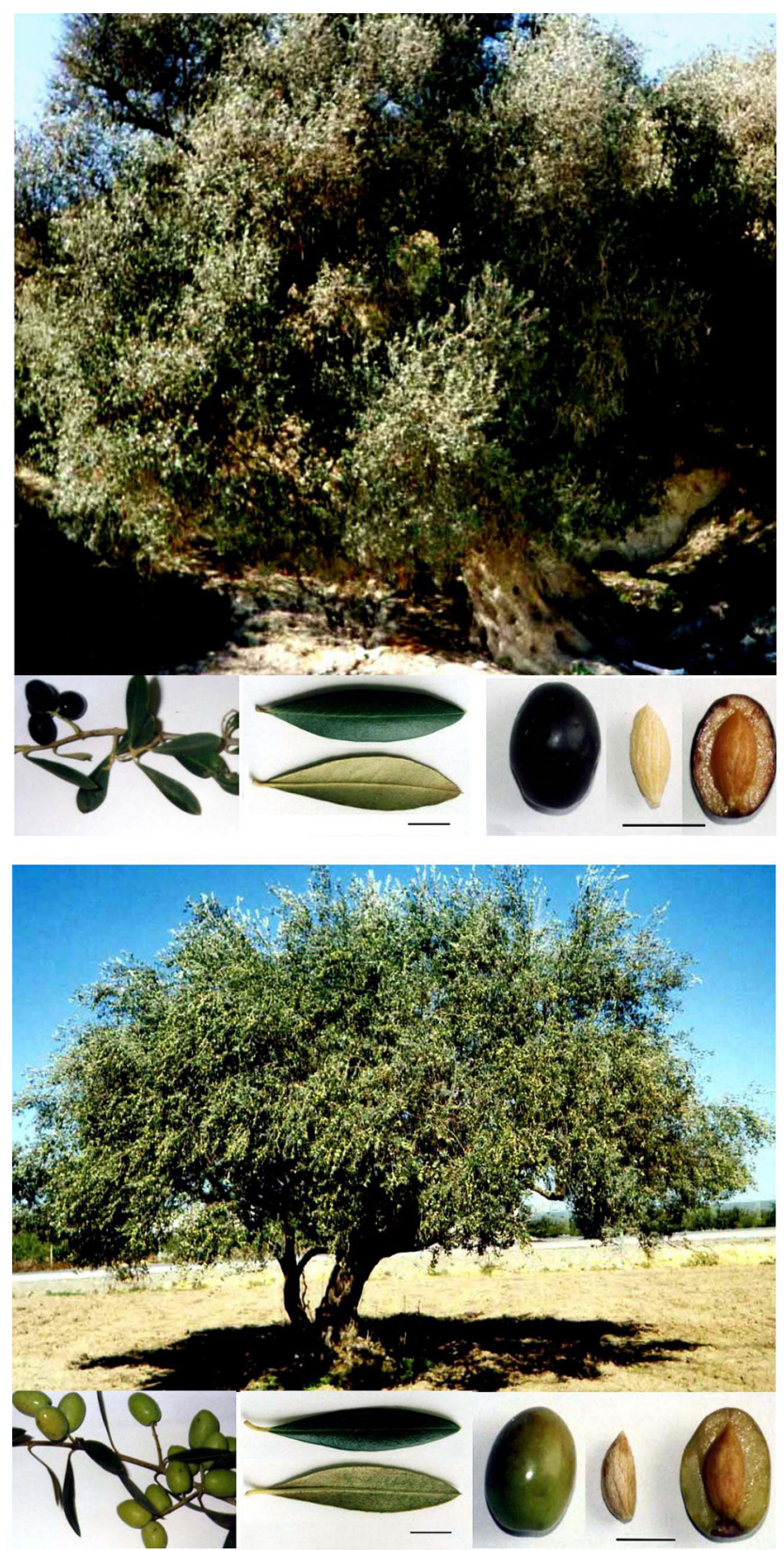

Fig. 1. Olive trees. Wild (above) and cultivated (below). Branch with fruits, leaves, fruits and seeds. Bar represents $1 \mathrm{~cm}$

Olea europaea trees spread in the Mediterranean Basin where they are indigenous as well as in other regions with a Mediterranean climate where they have been introduced (Costa, 1998). All cultivated and wild olive trees belong to two botanical varieties: The wild olive trees or oleaster correspond to Olea europaea subsp. europaea L. var. sylvestris. The cultivated olive trees correspond to Olea europaea subsp. europaea L. var. europaea (Green, 2002) and comprise olive cultivars grown for oil or table olives. In addition to these two olive forms, a third form resembling oleasters by their physiognomy, has escaped from cultivation, and has been called feral form (Besnard et al., 2001; Mekuria et al., 2002; Breton et al., 2006a).
The description of oleaster and olive cultivars and/or the relationships between them were conducted using several markers as morphological traits (Bartolini et al., 1998; Rotondi et al., 2003; Idrissi \& Ouazzani, 2003; Hannachi et al., 2008a,b; Belaj et al., 2011), oil criteria (Baccouri et al., 2011; Dabbou et al., 2011; Krichene et al., 2007; Hannachi et al., 2013) and molecular markers (Belaj et al., 2007; Belaj et al., 2010; Hannachi et al., 2010; Erre et al., 2010; Belaj et al., 2011; Noormohammadi et al., 2014).

Several historical reports pointed out that Oleaster forests are native in Tunisia (Camps-Fabrer, 1997). Based on nuclear and cytoplasmic SSR markers, the indigenous and feral types have been noted in Tunisia both in natural and agro-ecosystems (Hannachi et al., 2008b, Hannachi et al., 2010). The modern Tunisian oleasters seem to issue from one refuge area (Breton et al., 2006a). In addition, gene flow continued between oleaster and cultivated olive trees (Lumaret \& Ouazzani, 2001) because several widespread olive cultivars are male sterile (Besnard et al., 2000; Hannachi \& Marzouk, 2012). In the case of olive cultivars in orchards, they may be pollinated by surrounding oleasters as reported in several countries (France, Algeria, Morocco, Tunisia and Spain). However, this crossing has no consequence on the oil quality which is determined by the maternal tissue of the fruit. Therefore such hybrid fruits may lead again to feral trees (Breton et al., 2005). Therefore, the use of wild olive trees in breeding programs has been proposed as an alternative approach to increase the diversity of genetic resources such as for example in Spain (Belaj et al., 2010). To attempt this aim, in situ and ex situ characterization of olive genotypes is crucial using several descriptors. In addition, the characterization of wild and cultivated olive trees is important to preserve the genetic diversity, to select parent for genetic crossing.

Seed morphology is an interesting tool in the description of plant families, species and varieties. Morphological analysis based in the comparison with geometric objects is useful to characterize the seeds in plant families (Cervantes et al., 2016) and for the determination of taxonomic relationships of species and subspecies. The shape of seeds in model species was quantified by comparison with a cardioid (Cervantes et al., 2010; Cervantes et al., 2012) thus allowing to accurately measure changes in seed shape in mutants, during development or in response to environmental conditions or stress situations (Martín et al., 2014). In addition comparison of seed images with geometric figures allows the identification of features between subspecies and varieties (Saadaoui et al., 2013), changes to growth conditions (Saadaoui et al., 2015) or particular climatic adaptations (Martín Gómez et al., 2016). In general, seed shape has less variation than size, but 
both magnitudes are independent and valuable in phenotyping.

In Tunisia, the olive, existing in a wild and cultivated state, is characterized by a high genetic variability. This work aims to find new phenotypic descriptors, which allowed us to better discriminate the varieties and cultivars. Stone size and shape were analyzed for multiple sites, in different bioclimatic zones of the country.

\section{Material and methods}

\section{Plant material}

Stones (seeds) were collected from 36 cultivated trees belonging to a total of 19 cultivars and from 55 wild olive trees, making a total of 91 trees (Table 1). Main characteristics of geographical sites are shown in Tables 1 and 2, geographical locations in Fig. 2. Samples comprise ten stones of each olive tree.

\section{Seed scanning}

Seeds were scanned with a CanoScanLiDE 110 with resolution set at 300 ppp horizontal and 300 ppp vertical. Each image contains ten seeds of each tree, thus the analysis includes 91 images of ten seeds each (Fig. 3).

\section{Image analysis}

Image analysis was done with Image (Ferreira \& Wayne, 2010) recording automatically the values of seed length $(\mathrm{cm})$, seed width $(\mathrm{cm})$, perimeter $(\mathrm{cm})$, area $\left(\mathrm{cm}^{2}\right)$, circularity index and roundness.

Circularity Index (Cox, 1927; Riley, 1941; Schwartz, 1980) or form factor (Rovner \& Gyulai, 2007) is defined as:

$$
I=\frac{4 \pi \times \text { area }}{\text { perimeter }^{2}}
$$

Roundness (Rovner \& Gyulai, 2007) is defined as:

$$
R=\frac{4 \mathrm{x} \text { area }}{\pi[\text { Major axis }]^{2}}
$$

Circularity Index depends on the perimeter, while Roundness depends on the major axis. Thus, in objects of uneven or rough surface, the values of the former decrease very rapidly with the oscillations in the surface. The ratio between Circularity Index and Roundness is given by:

$$
I / R=\frac{\pi^{2}[\text { Major axis }]^{2}}{\text { perimeter }^{2}}
$$

In consequence increases in the perimeter of a figure, maintaining the major axis constant or under low change, results in reduced circularity values relative to roundness.

\section{Statistical analysis}

The program SPSS v. 21 was used for the ANOVA with Scheffé test (different sample number between populations and bioclimatic regions), T Student test and Pearson correlation.

Principal Component Analysis (PCA) was conducted on wild and cultivated plants based on stone size and shape data. The hierarchical classification was also done using Euclidean Distance and Ward as the aggregation method. Analyses were computed using the software Statistica (Sta Soft Inc., Johannesburg, ZA).

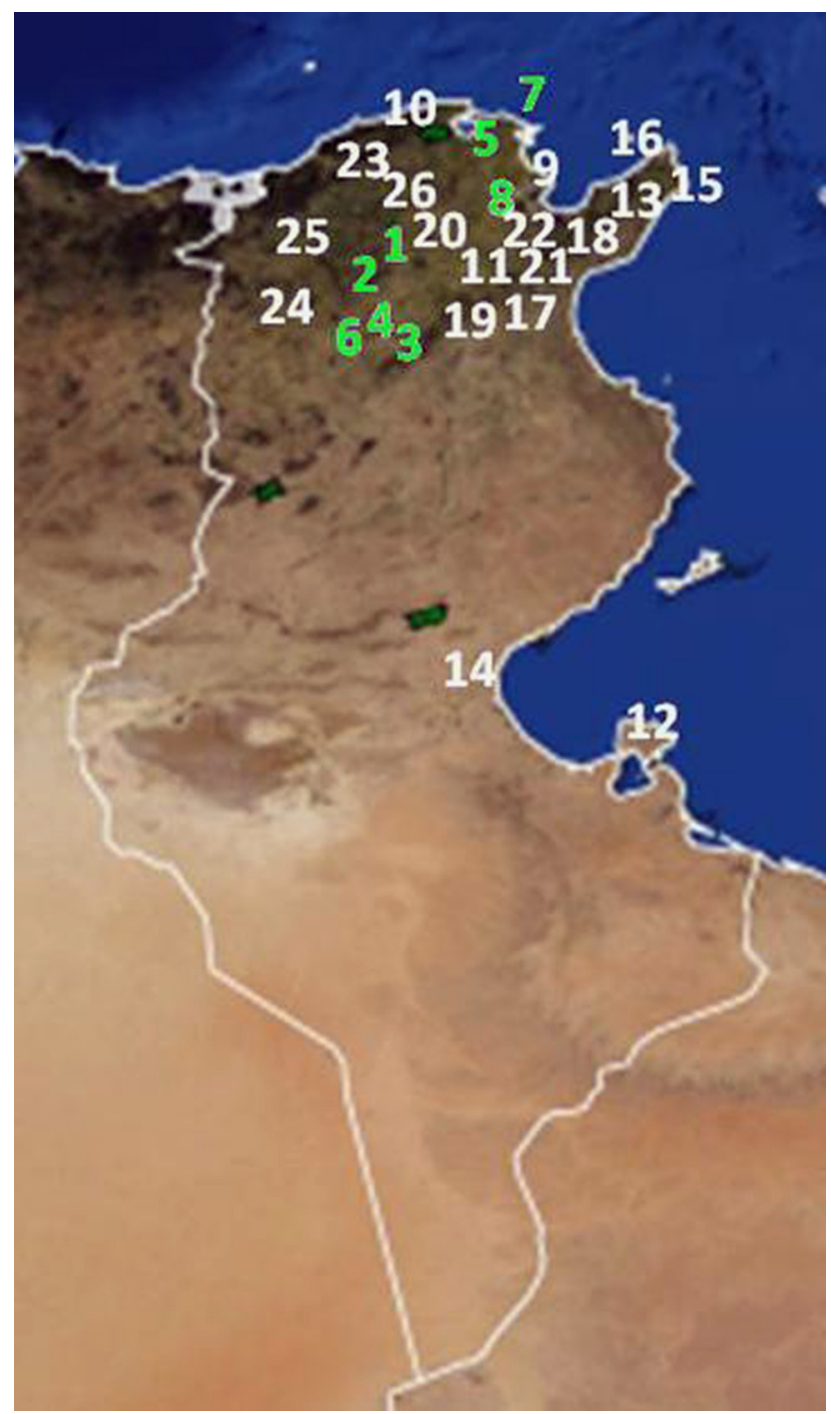

Fig. 2. Geographical sites of studied cultivated and wild olive trees. Green numbers (1 to 8 ) represent localities that have wild and cultivated populations. In white numbers (9 to 26), localities with only cultivated populations 
Table 1. Codes and localities of wild (oleaster) and cultivated olive trees

\begin{tabular}{|c|c|c|c|c|c|c|c|}
\hline $\begin{array}{c}\text { Olive Tree } \\
\text { cultivar }\end{array}$ & Code & Locality & Province & Olive Tree & Code & Locality & Province \\
\hline Chétoui & $\mathrm{C} 1$ & Slouguia & Béja & Oleaster & $\mathrm{O} 10$ & Zaghouan & Zaghouan \\
\hline Chétoui & $\mathrm{C} 2$ & Laaroussa & Siliana & Oleaster & O11 & Zaghouan & Zaghouan \\
\hline Chétoui & $\mathrm{C} 3$ & Téboursouk & Béja & Oleaster & $\mathrm{O} 12$ & Zaghouan & Zaghouan \\
\hline Chétoui & $\mathrm{C} 4$ & El-Alia & Bizerte & Oleaster & $\mathrm{O} 13$ & Zaghouan & Zaghouan \\
\hline Marsaline & $\mathrm{C} 5$ & Slouguia & Béja & Oleaster & O14 & Djerba & Medenine \\
\hline Marsaline & $\mathrm{C} 6$ & Laaroussa & Siliana & Oleaster & O15 & Azmour & Haouaria \\
\hline Chaïbi & $\mathrm{C} 7$ & Testour & Béja & Oleaster & O16 & Zaghouan & Zaghouan \\
\hline Chaïbi & $\mathrm{C} 8$ & Téboursouk & Béja & Oleaster & $\mathrm{O} 17$ & Zaghouan & Zaghouan \\
\hline Chaïbi & C9 & Laaroussa & Siliana & Oleaster & O18 & Azmour & Kélibia \\
\hline Chaïbi & $\mathrm{C} 10$ & Tébousouk & Béja & Oleaster & O19 & Azmour & Kélibia \\
\hline Chemlali & $\mathrm{C} 11$ & Slouguia & Béja & Oleaster & $\mathrm{O} 20$ & Chenenni & Gabès \\
\hline Meski & $\mathrm{C} 12$ & EL-Alia & Bizerte & Oleaster & $\mathrm{O} 21$ & Azmour & Kélibia \\
\hline Meski & $\mathrm{C} 13$ & Dougga & Béja & Oleaster & $\mathrm{O} 22$ & Azmour & Kélibia \\
\hline Meski & $\mathrm{C} 14$ & Slouguia & Béja & Oleaster & $\mathrm{O} 23$ & Testour & Béja \\
\hline Meski & $\mathrm{C} 15$ & El-Alia & Bizerte & Oleaster & $\mathrm{O} 24$ & Testour & Béja \\
\hline Besbessi & $\mathrm{C} 16$ & Laaroussa & Siliana & Oleaster & $\mathrm{O} 25$ & Belvedère & Tunis \\
\hline Besbessi & $\mathrm{C} 17$ & Testour & Béja & Oleaster & $\mathrm{O} 26$ & Ras-Jbel & Bizerte \\
\hline Besbessi & $\mathrm{C} 18$ & Slouguia & Béja & Oleaster & $\mathrm{O} 27$ & Slouguia & Béja \\
\hline Berboui & C19 & Laaroussa & Siliana & Oleaster & $\mathrm{O} 28$ & Téboursouk & Béja \\
\hline Gerboui & $\mathrm{C} 20$ & Téboursouk & Béja & Oleaster & $\mathrm{O} 29$ & Ichkeul & Bizerte \\
\hline Gerboui & $\mathrm{C} 21$ & Téboursouk & Béja & Oleaster & $\mathrm{O} 30$ & Ichkeul & Bizerte \\
\hline Gerboui & $\mathrm{C} 22$ & Laaroussa & Siliana & Oleaster & O31 & Ichkeul & Bizerte \\
\hline Gerboui & $\mathrm{C} 23$ & Dougga & Béja & Oleaster & $\mathrm{O} 32$ & Ichkeul & Bizerte \\
\hline Gerboui & $\mathrm{C} 24$ & Téboursouk & Béja & Oleaster & $\mathrm{O} 33$ & Kélibia & Kélibia \\
\hline Oueslati & $\mathrm{C} 25$ & Téboursouk & Béja & Oleaster & $\mathrm{O} 34$ & Ech-Raf & Haouaria \\
\hline Rajou & $\mathrm{C} 26$ & Ras-Jbel & Bizerte & Oleaster & $\mathrm{O} 35$ & Ech-Raf & Haouaria \\
\hline Zayati & $\mathrm{C} 27$ & Laaroussa & Siliana & Oleaster & O36 & Ech-Raf & Haouaria \\
\hline $\mathrm{Nib}$ & $\mathrm{C} 28$ & Ras-Jbel & Bizerte & Oleaster & $\mathrm{O} 37$ & El-Alia & Bizerte \\
\hline Zarras & $\mathrm{C} 29$ & Téboursouk & Béja & Oleaster & $\mathrm{O} 38$ & Saouaf & Zaghouan \\
\hline Sayali & $\mathrm{C} 30$ & Slouguia & Béja & Oleaster & O39 & Jbel-Abderahmen & Nabeul \\
\hline Beldi & $\mathrm{C} 31$ & Téboursouk & Béja & Oleaster & $\mathrm{O} 40$ & Kélibia & Kélibia \\
\hline Neb Jmel & $\mathrm{C} 32$ & Testour & Béja & Oleaster & O41 & Dhra-Joudar & Zagouah \\
\hline Awam & $\mathrm{C} 33$ & Dougga & Béja & Oleaster & $\mathrm{O} 42$ & Mjez El Bab & Béja \\
\hline Chemlali & $\mathrm{C} 34$ & Tunis & Tunis & Oleaster & $\mathrm{O} 43$ & Jbel-Abderahmen & Nabeul \\
\hline Roumi & $\mathrm{C} 35$ & Téboursouk & Béja & Oleaster & O44 & Saouaf & Zaghouan \\
\hline Limi & $\mathrm{C} 36$ & Teboursouk & Béja & Oleaster & $\mathrm{O} 45$ & Zriba & Zaghouan \\
\hline Oleaster & $\mathrm{O} 1$ & Ras-Jbel & Bizerte & Oleaster & O46 & Zaghouan & Zaghouan \\
\hline Oleaster & $\mathrm{O} 2$ & Dougga & Béja & Oleaster & $\mathrm{O} 47$ & Jbel West & Zaghouan \\
\hline Oleaster & $\mathrm{O} 3$ & Belvedère & Tunis & Oleaster & $\mathrm{O} 48$ & Tunis & Tunis \\
\hline Oleaster & $\mathrm{O} 4$ & Belvedère & Tunis & Oleaster & O49 & Sejnan & Bizerte \\
\hline Oleaster & O5 & Testour & Béja & Oleaster & O50 & Borj-Messaoudi & El-Kef \\
\hline Oleaster & O6 & Slouguia & Béja & Oleaster & O51 & Tbaba & Jendouba \\
\hline Oleaster & O7 & Ichkeul & Bizerte & Oleaster & O52 & Mateur & Bizerte \\
\hline Oleaster & O8 & Ichkeul & Bizerte & Oleaster & $\mathrm{O} 53$ & Tunis & Tunis \\
\hline \multirow[t]{2}{*}{ Oleaster } & O9 & Ichkeul & Bizerte & Oleaster & O54 & Laaroussa & Siliana \\
\hline & & & & Oleaster & $\mathrm{O} 55$ & Laaroussa & Siliana \\
\hline
\end{tabular}

C: Cultivated olive tree; O: Oleaster (wild olive tree).

\section{Results}

\section{Size and shape comparison between stones obtained from the wild plants}

Stone size and shape showed high variability both in wild and cultivated olive trees. In wild trees seed image area oscillated between 0.12 and $0.74 \mathrm{~cm}^{2}$ with mean values of $0.38 \mathrm{~cm}^{2}$ and standard deviation of $0.11 \mathrm{~cm}^{2}$ (Table 3). Roundness oscillated between 0.37 and 0.93 with mean values of 0.53 and standard deviation of 0.08 . Circularity oscillated between 0.51 and 0.84 with mean values of 0.69 and standard deviation of 0.05 . The Pearson correlation values 
Table 2. Main characteristics of localities of studied wild and cultivated olive trees

\begin{tabular}{|c|c|c|c|c|c|}
\hline Locality & Code & Latitude & Longitude & $\begin{array}{l}\text { Altitude } \\
(\mathrm{m})\end{array}$ & Bioclimate \\
\hline Slouguia & 1 & $36^{\circ} 36^{\prime} 37.81^{\prime \prime} \mathrm{N}$ & 09³1'53.39"'E & 68 & Sub-humid \\
\hline Testour & 2 & $36^{\circ} 33^{\prime} 06,92^{\prime \prime} \mathrm{N}$ & $09^{\circ} 26^{\prime} 58,96^{\prime \prime} \mathrm{E}$ & 93 & Sub-humid \\
\hline Laaroussa & 3 & $36^{\circ} 22^{\prime} 51,94^{\prime \prime} \mathrm{N}$ & $09^{\circ} 27^{\prime} 16,37^{\prime \prime} \mathrm{E}$ & 197 & Semi-arid \\
\hline Téboursouk & 4 & $36^{\circ} 27^{\prime} 40.60^{\prime \prime} \mathrm{N}$ & $09^{\circ} 14^{\prime} 47.48^{\prime \prime} \mathrm{E}$ & 472 & Sub-humid \\
\hline El-Alia & 5 & $37^{\circ} 10^{\prime} 38.70^{\prime \prime} \mathrm{N}$ & $10^{\circ} 02^{\prime} 05.21^{\prime \prime} \mathrm{E}$ & 142 & Sub-humid \\
\hline Dougga & 6 & $36^{\circ} 24^{\prime} 02.75^{\prime \prime} \mathrm{N}$ & $09^{\circ} 14^{\prime} 14.75^{\prime \prime} \mathrm{E}$ & 380 & Sub-humid \\
\hline Ras-Jbel & 7 & $37^{\circ} 11^{\prime} 59.56^{\prime \prime} \mathrm{N}$ & $10^{\circ} 07^{\prime} 28.84^{\prime \prime} \mathrm{E}$ & 82 & Sub-humid \\
\hline Tunis & 8 & $36^{\circ} 49^{\prime} 12.99^{\prime \prime} \mathrm{N}$ & $10^{\circ} 10^{\prime} 13.97^{\prime \prime} \mathrm{E}$ & 25 & Upper semi-arid \\
\hline Belvedère & 9 & $36^{\circ} 49^{\prime} 21.27^{\prime \prime} \mathrm{N}$ & $10^{\circ} 10^{\prime} 23.83^{\prime \prime} \mathrm{E}$ & 43 & Upper Semi-arid \\
\hline Ichkeul & 10 & $37^{\circ} 07^{\prime} 15.22^{\prime \prime} \mathrm{N}$ & $09^{\circ} 38^{\prime} 55.91^{\prime \prime} \mathrm{E}$ & 204 & Sub-humid \\
\hline Zaghouan & 11 & $36^{\circ} 24^{\prime} 00.81^{\prime \prime} \mathrm{N}$ & $10^{\circ} 09^{\prime} 29.77^{\prime \prime} \mathrm{E}$ & 177 & Upper Semi-arid \\
\hline Djerba & 12 & $33^{\circ} 49^{\prime} 25.53^{\prime \prime} \mathrm{N}$ & $10^{\circ} 50^{\prime} 46.72^{\prime \prime} \mathrm{E}$ & 19 & Arid \\
\hline Azmour & 13 & $36^{\circ} 55^{\prime} 28.18^{\prime \prime} \mathrm{N}$ & $11^{\circ} 00^{\prime} 24.88^{\prime \prime} \mathrm{E}$ & 56 & Sub-humid \\
\hline Chenenni & 14 & $33^{\circ} 54^{\prime} 05.05^{\prime \prime} \mathrm{N}$ & $10^{\circ} 05^{\prime} 20.62^{\prime \prime} \mathrm{E}$ & 13 & Arid \\
\hline Kélibia & 15 & $36^{\circ} 51^{\prime} 49.67^{\prime \prime} \mathrm{N}$ & $11^{\circ} 05^{\prime} 58.48^{\prime \prime} \mathrm{E}$ & 12 & Sub-humid \\
\hline Ech-Raf & 16 & $36^{\circ} 59^{\prime} 57.92^{\prime \prime} \mathrm{N}$ & $11^{\circ} 02^{\prime} 12.69^{\prime \prime} \mathrm{E}$ & 32 & Sub-humid \\
\hline Saouaf & 17 & $36^{\circ} 11^{\prime} 47.54^{\prime \prime} \mathrm{N}$ & $10^{\circ} 11^{\prime} 33.63^{\prime \prime} \mathrm{E}$ & 199 & Upper Semi-arid \\
\hline Jbel-Abderahmen & 18 & $36^{\circ} 44^{\prime} 21.79^{\prime \prime} \mathrm{N}$ & $10^{\circ} 41^{\prime} 26.58^{\prime \prime} \mathrm{E}$ & 340 & Upper Semi-arid \\
\hline Dhra-Joudar & 19 & $36^{\circ} 16^{\prime} 25.20^{\prime \prime} \mathrm{N}$ & $10^{\circ} 01^{\prime} 14.77^{\prime \prime} \mathrm{E}$ & 358 & Upper Semi-arid \\
\hline Mjez El Bab & 20 & $36^{\circ} 38^{\prime} 44.87^{\prime \prime} \mathrm{N}$ & $09^{\circ} 36^{\prime} 15.34^{\prime \prime} \mathrm{E}$ & 55 & Upper Semi-arid \\
\hline Zriba & 21 & $36^{\circ} 20^{\prime} 19.66^{\prime \prime} \mathrm{N}$ & $10^{\circ} 11^{\prime} 33.71^{\prime \prime} \mathrm{E}$ & 186 & Upper Semi-arid \\
\hline Jbel West & 22 & $36^{\circ} 32^{\prime} 08.64^{\prime \prime} \mathrm{N}$ & $10^{\circ} 04^{\prime} 26.91^{\prime \prime} \mathrm{E}$ & 136 & Upper Semi-arid \\
\hline Sejnan & 23 & $37^{\circ} 03^{\prime} 07.55^{\prime \prime} \mathrm{N}$ & $09^{\circ} 14^{\prime} 31.94^{\prime \prime} \mathrm{E}$ & 195 & Sub-humid \\
\hline Borj-Messaoudi & 24 & $36^{\circ} 12^{\prime} 00.64^{\prime \prime} \mathrm{N}$ & $08^{\circ} 44^{\prime} 41.48^{\prime \prime} \mathrm{E}$ & 720 & Sub-humid \\
\hline Tbaba & 25 & $36^{\circ} 30^{\prime} 28.66^{\prime \prime} \mathrm{N}$ & $08^{\circ} 39^{\prime} 53.02^{\prime \prime} \mathrm{E}$ & 501 & Sub-humid \\
\hline Mateur & 26 & $37^{\circ} 02^{\prime} 32.83^{\prime \prime} \mathrm{N}$ & $09^{\circ} 40^{\prime} 10.48^{\prime \prime} \mathrm{E}$ & 32 & Sub-humid \\
\hline
\end{tabular}
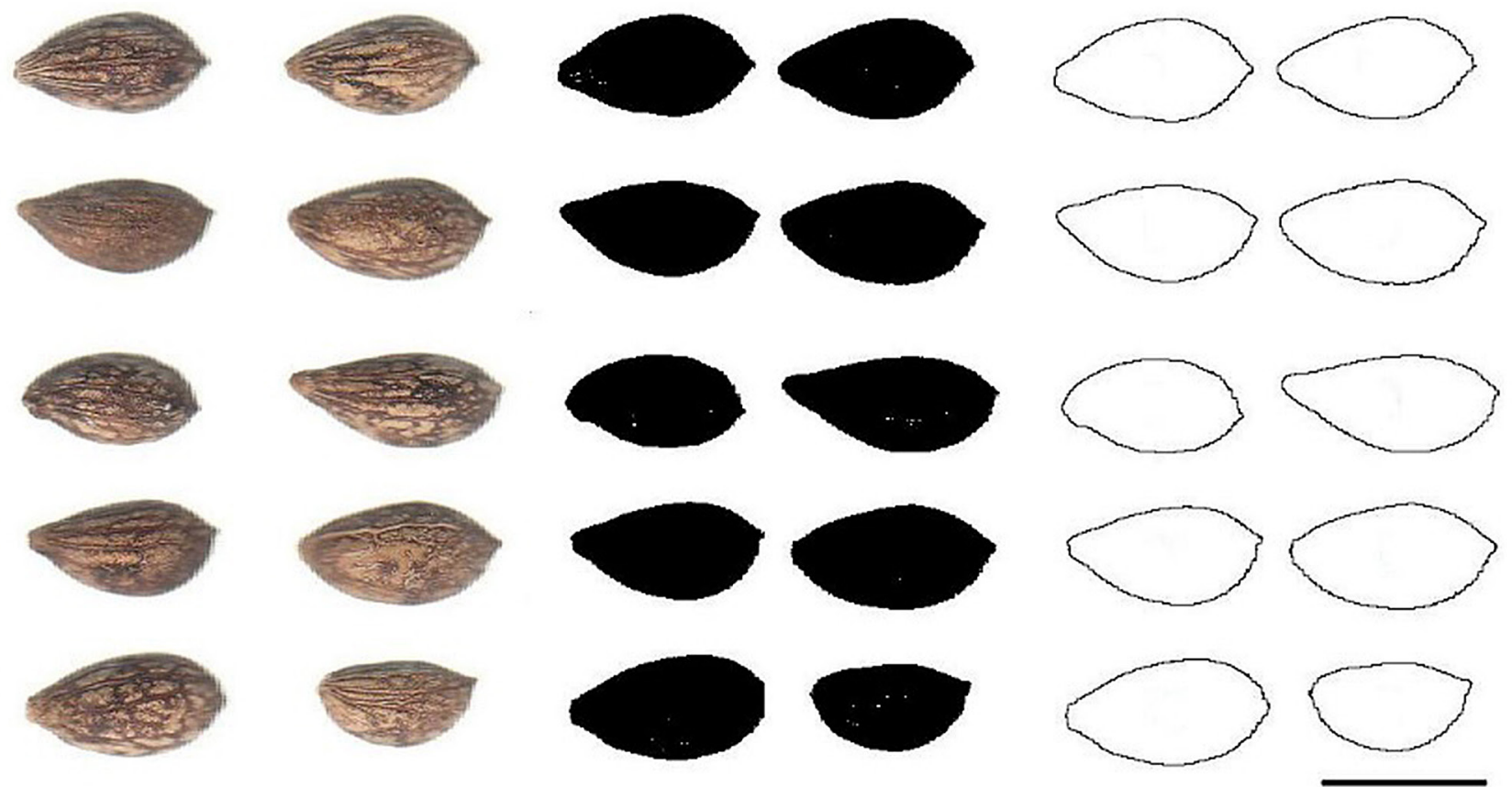

Fig. 3. A summary of the method used to quantify stone size and shape. Left: Stone images obtained by scan. Center: Profiles corresponding to each image. Right: Silhouettes. The program calculates: length, perimeter, area, circularity index and roundness index from the silhouettes 
Table 3. Values of magnitudes related with size and shape in Olea europea seeds from wild and cultivated plants and results of $\mathrm{T}$ test comparing both groups

\begin{tabular}{|c|c|c|c|c|c|c|c|c|}
\hline & & $\mathrm{N}$ & Mean & Min & Max & $\begin{array}{l}\text { Standard } \\
\text { Deviation }\end{array}$ & $\begin{array}{c}\text { Standard } \\
\text { error mean }\end{array}$ & $\begin{array}{c}\text { Bilateral } \\
\text { sig. }\end{array}$ \\
\hline \multirow{2}{*}{ Area } & wild & 550 & 0.38 & 0.12 & 0.74 & 0.11 & 0.00 & \multirow{2}{*}{0.000} \\
\hline & cultivated & 359 & 0.75 & 0.40 & 1.47 & 0.21 & 0.01 & \\
\hline \multirow{2}{*}{ Roundness } & wild & 550 & 0.53 & 0.37 & 0.93 & 0.08 & 0.00 & \multirow{2}{*}{0.003} \\
\hline & cultivated & 359 & 0.55 & 0.30 & 0.85 & 0.11 & 0.01 & \\
\hline \multirow{2}{*}{ Circularity } & wild & 550 & 0.69 & 0.51 & 0.84 & 0.05 & 0.00 & \multirow{2}{*}{0.000} \\
\hline & cultivated & 359 & 0.66 & 0.46 & 0.79 & 0.05 & 0.00 & \\
\hline \multirow{2}{*}{ Length } & wild & 550 & 1.01 & 0.52 & 1.52 & 0.20 & 0.01 & \multirow{2}{*}{0.000} \\
\hline & cultivated & 359 & 1.39 & 0.87 & 2.12 & 0.23 & 0.01 & \\
\hline \multirow{2}{*}{ Width } & wild & 550 & 0.52 & 0.31 & 0.73 & 0.07 & 0.00 & \multirow{2}{*}{0.000} \\
\hline & cultivated & 359 & 0.73 & 0.48 & 1.12 & 0.12 & 0.01 & \\
\hline \multirow{2}{*}{ Length/Width } & wild & 550 & 1.92 & 1.08 & 2.72 & 0.28 & 0.01 & \multirow{2}{*}{0.047} \\
\hline & cultivated & 359 & 1.88 & 1.18 & 3.32 & 0.35 & 0.02 & \\
\hline
\end{tabular}

between seed area and roundness or seed area and circularity were negative, i.e., larger seeds had lower values of roundness and circularity (Table 4 ).

\section{Size and shape comparison between stones obtained from the cultivated olive trees}

In the cultivated plants, the seed image area oscillated between $0.40 \mathrm{~cm}^{2}$ and $1.47 \mathrm{~cm}^{2}$ with mean values of $0.75 \mathrm{~cm}^{2}$ and standard deviation of $0.21 \mathrm{~cm}^{2}$ (Table 3). Roundness oscillated between 0.30 and 0.85 with mean values of 0.55 and standard deviation of 0.11 , and circularity oscillated between 0.46 and 0.79 with mean values of 0.66 and standard deviation of 0.05 . The Pearson correlation values between seed area and circularity were negative. The Pearson correlation values between seed area and roundness were not significant. Larger seeds had lower values of circularity, but not of roundness (Table 4).

\section{Climatic effect in stone size and shape and local differences in wild plants}

Larger seed size in arid regions and smaller in sub-humid, upper semi-arid and semi-arid regions (Table 5a), may be the result of larger seeds in the two trees used in the arid region and not specific effect of climate on stone size. Roundness was higher in the semi-arid region and lower in the arid region with intermediate values in the other two regions (sub-humid and upper semi-arid). Similar results were obtained for circularity index. Relationships between roundness and circularity values in the climatic regions were conserved, although with lower values in roundness.

Table 4. Values of Person Correlation. **:Significant at level 0,01 (bilateral) ; *:Significant at level 0,05 (bilateral)

\begin{tabular}{|c|c|c|c|c|c|c|c|}
\hline \multirow{8}{*}{ 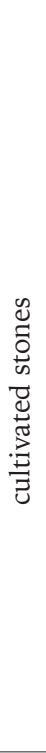 } & & \multicolumn{6}{|c|}{ wild stones } \\
\hline & & Area & Roundness & Circularity & Length & Width & Length/Width \\
\hline & Area & 1.000 & $\begin{array}{l}-.441^{* *} \\
\text { sig. bilateral } \\
(0.000)\end{array}$ & $\begin{array}{l}-.446^{* *} \\
\text { sig. bilateral } \\
(0.000)\end{array}$ & $\begin{array}{l}.916^{* *} \\
\text { sig. bilateral } \\
(0.000)\end{array}$ & $\begin{array}{l}.862^{* *} \\
\text { sig. bilateral } \\
(0.000)\end{array}$ & $\begin{array}{l}.459^{* *} \\
\text { sig. bilateral } \\
(0.000)\end{array}$ \\
\hline & roundness & $\begin{array}{l}-0.038 \\
\text { sig. bilateral } \\
(0.474)\end{array}$ & 1.000 & $\begin{array}{l}.766^{* *} \\
\text { sig. bilateral } \\
(0.000)\end{array}$ & $\begin{array}{l}-.736^{* *} \\
\text { sig. bilateral } \\
(0.000)\end{array}$ & $\begin{array}{l}-0.009 \\
\text { sig. bilateral } \\
(0.832)\end{array}$ & $\begin{array}{l}-.979 * * \\
\text { sig. bilateral } \\
(0.000)\end{array}$ \\
\hline & circularity & $\begin{array}{l}-.404^{* *} \\
\text { sig. bilateral } \\
(0.000)\end{array}$ & $\begin{array}{l}.633^{* *} \\
\text { sig. bilateral } \\
(0.000\end{array}$ & 1.000 & $\begin{array}{l}-.676^{* *} \\
\text { sig. bilateral } \\
(0.000)\end{array}$ & $\begin{array}{l}-.134^{* *} \\
\text { sig. bilateral } \\
(0.002)\end{array}$ & $\begin{array}{l}-.792^{* *} \\
\text { sig. bilateral } \\
(0.000)\end{array}$ \\
\hline & Length & $\begin{array}{l}.763^{* *} \\
\text { sig. bilateral } \\
(0.000)\end{array}$ & $\begin{array}{l}-.635^{* *} \\
\text { sig. bilateral } \\
(0.000)\end{array}$ & $\begin{array}{l}-.741^{* *} \\
\text { sig. bilateral } \\
(0.000)\end{array}$ & 1.000 & $\begin{array}{l}.632^{* *} \\
\text { sig. bilateral } \\
(0.000)\end{array}$ & $\begin{array}{l}.756^{* *} \\
\text { sig. bilateral } \\
(0.000)\end{array}$ \\
\hline & Width & $\begin{array}{l}.843^{* *} \\
\text { sig. bilateral } \\
(0.00\end{array}$ & $\begin{array}{l}.476^{* *} \\
\text { sig. bilateral } \\
(0.000)\end{array}$ & $\begin{array}{l}-0.036 \\
\text { sig. bilateral } \\
(0.502)\end{array}$ & $\begin{array}{l}.335^{* *} \\
\text { sig. bilateral } \\
(0.000\end{array}$ & 1.000 & $\begin{array}{l}0.022 \\
\text { sig. bilateral } \\
(0.604)\end{array}$ \\
\hline & length /width & $\begin{array}{l}0.010 \\
\text { sig. bilateral } \\
(0.848)\end{array}$ & $\begin{array}{l}-.967^{* *} \\
\text { sig. bilateral } \\
(0.000)\end{array}$ & $\begin{array}{l}-.702^{* *} \\
\text { sig. bilateral } \\
(0.000\end{array}$ & $\begin{array}{l}.629^{* *} \\
\text { sig. bilateral } \\
(0.000)\end{array}$ & $\begin{array}{l}-.490 * * \\
\text { sig. bilateral } \\
(0.000)\end{array}$ & 1.000 \\
\hline
\end{tabular}


Table 5. Comparison of size and shape in wild Olea europaea stones by climatic regions (a) and by localities in the climatic regions (b). $\mathrm{N}$ is the number of seeds analyzed in each case. The same letter ( $, \mathrm{b}, \mathrm{c}$ ) in the superindex for a given a) column indicates no difference between bioclimates or localities in the results from one factor Anova

a)

\begin{tabular}{lccccccc}
\hline \multicolumn{1}{c}{ BioClimate } & $\mathrm{N}$ & Area & Roundness & Circularity & Length & Width & Length/Width \\
\hline Sub-humid & 312 & $0.39^{\mathrm{a}}$ & $0.53^{\mathrm{b}}$ & $0.68^{\mathrm{a}, \mathrm{b}}$ & $1.03^{\mathrm{b}}$ & $0.52^{\mathrm{a}}$ & $1.97^{\mathrm{b}}$ \\
Semi-arid superior & 198 & $0.35^{\mathrm{a}}$ & $0.54^{\mathrm{b}}$ & $0.70^{\mathrm{b}}$ & $0.97^{\mathrm{a}, \mathrm{b}}$ & $0.50^{\mathrm{a}}$ & $1.93^{\mathrm{b}}$ \\
Semi-arid & 20 & $0.35^{\mathrm{a}}$ & $0.62^{\mathrm{c}}$ & $0.73^{\mathrm{c}}$ & $0.90^{\mathrm{a}}$ & $0.54^{\mathrm{a}}$ & $1.67^{\mathrm{a}}$ \\
Arid & 20 & $0.49^{\mathrm{b}}$ & $0.46^{\mathrm{a}}$ & $0.66^{\mathrm{a}}$ & $1.22^{\mathrm{c}}$ & $0.54^{\mathrm{a}}$ & $2.25^{\mathrm{c}}$ \\
\hline
\end{tabular}

b)

\begin{tabular}{|c|c|c|c|c|}
\hline \multirow{2}{*}{ Locality } & \multirow{2}{*}{$\mathrm{N}$} & \multicolumn{3}{|c|}{ Sub-Humid } \\
\hline & & Area & Roundness & Circularity \\
\hline Azmour & 50 & $0.39 \mathrm{a} a, \mathrm{~b}, \mathrm{c}$ & $0.54^{a, b, c}$ & $0.66^{a, b}$ \\
\hline Borj-Messaoudi & 10 & $0.33^{a, b}$ & $0.58^{b, c}$ & $0.72^{a, b}$ \\
\hline Dougga & 10 & $0.51^{c, d}$ & $0.63^{c}$ & $0.70^{a, b}$ \\
\hline Ech-Raf & 32 & $0.45^{b, c, d}$ & $0.48^{a, b}$ & $0.68^{a, b}$ \\
\hline El-Alia & 10 & $0.31^{a}$ & $0.63^{c}$ & $0.71^{a, b}$ \\
\hline Ichkeul & 70 & $0.31^{a}$ & $0.52^{a, b, c}$ & $0.69 \mathrm{a}, \mathrm{b}$ \\
\hline Kélibia & 20 & $0.57^{\mathrm{d}}$ & $0.50^{\mathrm{a}, \mathrm{b}}$ & $0.67^{a, b}$ \\
\hline Mateur & 10 & $0.43^{a, b, c}$ & $0.56^{a, b, c}$ & $0.71^{a, b}$ \\
\hline Ras-Jbel & 20 & $0.44^{\mathrm{a}, \mathrm{b}, \mathrm{c}, \mathrm{d}}$ & $0.45^{a}$ & $0.64^{a}$ \\
\hline Sejnan & 10 & $0.30^{a}$ & $0.50^{\mathrm{a}, \mathrm{b}}$ & $0.67 \mathrm{a}, \mathrm{b}$ \\
\hline Slouguia & 20 & $0.38^{a, b, c}$ & $0.50^{\mathrm{a}, \mathrm{b}}$ & $0.70^{a, b}$ \\
\hline Tbaba & 10 & $0.33^{\mathrm{a}, \mathrm{b}}$ & $0.56^{a, b, c}$ & $0.70^{a, b}$ \\
\hline Téboursouk & 10 & $0.32^{a, b}$ & $0.58^{b, c}$ & $0.73^{b}$ \\
\hline Testour & 30 & $0.43 a, b, c$ & $0.53 a, b, c$ & $0.70^{a, b}$ \\
\hline \multirow[t]{2}{*}{ Mean } & 312 & 0.39 & 0.53 & $0.68^{a}$ \\
\hline & \multicolumn{4}{|c|}{ Upper semi-arid } \\
\hline Belvedère & 30 & $0.32^{a, b}$ & $0.60^{b, c}$ & $0.73^{b, c}$ \\
\hline Dhra-Joudar & 10 & $0.24^{\mathrm{a}}$ & $0.61^{b, c}$ & $0.77^{c}$ \\
\hline Jbel West & 10 & $0.30^{a, b}$ & $0.51^{\mathrm{a}}$ & $0.67^{a}$ \\
\hline Jbel-Abderahmen & 20 & $0.31^{\mathrm{a}, \mathrm{b}}$ & $0.49^{a}$ & $0.69^{\mathrm{a}, \mathrm{b}}$ \\
\hline Mjez El Bab & 10 & $0.36^{\mathrm{a}, \mathrm{b}}$ & $0.66^{c}$ & $0.76^{c}$ \\
\hline Saouaf & 20 & $0.33^{\mathrm{a}, \mathrm{b}}$ & $0.53^{\mathrm{a}, \mathrm{b}}$ & $0.67^{a}$ \\
\hline Tunis & 20 & $0.28^{\mathrm{a}}$ & $0.63^{c}$ & $0.77^{c}$ \\
\hline Zaghouan & 68 & $0.4^{b}$ & $0.50^{a}$ & $0.67^{a}$ \\
\hline Zriba & 10 & $0.54^{\mathrm{c}}$ & $0.45^{\mathrm{a}}$ & $0.64^{\mathrm{a}}$ \\
\hline \multirow[t]{2}{*}{ Mean } & 198 & 0.35 & 0.541 & 0.70 \\
\hline & \multicolumn{4}{|c|}{ Semi-arid } \\
\hline Laaroussa (O54) & 10 & $0.34^{a}$ & $0.75^{b}$ & $0.77^{b}$ \\
\hline Laaroussa (O55) & 10 & $0.37^{b}$ & $0.5^{\mathrm{a}}$ & $0.69^{a}$ \\
\hline \multirow[t]{2}{*}{ Mean } & 20 & 0.35 & 0.63 & 0.73 \\
\hline & \multicolumn{4}{|c|}{ Arid } \\
\hline Djerba & 10 & $0.36^{a}$ & $0.50^{\mathrm{b}}$ & $0.68^{b}$ \\
\hline Chenenni & 10 & $0.61^{\mathrm{b}}$ & $0.43^{a}$ & $0.63^{a}$ \\
\hline Mean & 20 & 0.46 & 0.46 & 0.66 \\
\hline
\end{tabular}


Differences between localities in the sub-humid region were found for size (area) and shape (roundness and circularity, Table $5 \mathrm{~b}$ ). Area values oscillated between 0.30 (Sejnan) and $0.57 \mathrm{~cm}^{2}$ (Kélibia). Roundness values oscillated between 0.45 (Ras-Jbel) and 0.63 (Dougga and El-Alia) and circularity between 0.64 (Ras-Jbel) and 0.73 (Téboursouk).

In the upper semi-arid region, differences between localities were found for size (area) and shape (roundness and circularity; Table 5b). Area values oscillated between 0.24 (Dhra-Joudar) and $0.54 \mathrm{~cm}^{2}$ (Zriba). Roundness values oscillated between 0.45 (Zriba) and 0.66 (Mjez El Bab) and circularity between 0.64 (Zriba) and 0.77 (Dhra-Joudar and Tunis).

Comparison of size and shape between stones of two wild individuals from the same population in the semi-arid region revealed differences in seed size and shape (Table $5 b$ ).

\section{Differences between seeds from plants of diverse cultivars grown in different regions}

The cultivation of a variety in two different regions allows to investigate the size and shape of the seed in response to the climatic and geographic effect.

Seeds from cultivars Besbessi, Chaïbi, Chétoui, Gerboui and Marsaline where obtained both in sub-humid and semi-arid climates. Seeds from cultivar Chemlali where obtained from Sub-humid and Semi-arid superior climates. Although some of the cultivars presented a trend to increased seed area in the subhumid climate (Chaïbi, Chétoui, Marsaline, Chemlali; Table 6), this was not conserved in all cultivars. Roundness values were higher in the semi-arid regions in four of six varieties (Table 6).

\section{Size and shape comparison between stones obtained from wild and cultivated plants: mutivariate analysis}

Principal components analysis (PCA) reduced stone size and shape data to two principal components which accounted for $98.88 \%$ of the total variation (Table 7). The first component (PC1) accounted for $70.34 \%$ of the total variation and was mainly correlated negatively with area, perimeter and positively with circularity, length/width, and length $2 /$ perimeter $^{2}$. The second component PC2 explained $28.54 \%$ of the total variation and is defined positively with roundness and perimeter and negatively with length (Table 7). The dispersion of studied plants on the plan defined by PC1 and PC2 showed that wild plants were grouped at the upper right dial defined by circularity, length/width, lengh ${ }^{2} /$ perimeter $^{2}$, roundness, length and width. The cultivated plants were placed at the left dial (Fig. 4). The stones obtained from wild and cultivated plants revealed differences both in size and shape (Table 3). Stones from cultivated plants are larger and present lower values of circularity index and higher values of roundness than the stones from wild plants. However, the centre of PCA plot contains a mixed group of wild and cultivated olive trees (Fig. 4).

The hierarchical analysis was conducted based on stone data of studied plants (Fig. 5). The dendrogram obtained showed two cluster CL1 and CL2; each one was divided on two sub-clusters. In all four sub-clusters were identified (CL1/1, CL1/2, CL2/1 and CL2/2). Sub-cluster CL1/1 comprises only wild plants which were well separated from cultivated ones. Sub-cluster CL1/2 comprises 19 wild and three cultivated olive trees, C24 (Gerboui), C19 (Gerboui)

Table 6. Comparison of size and shape between seeds from plants of diverse cultivars grown in different regions

\begin{tabular}{|c|c|c|c|c|c|c|c|c|}
\hline $\begin{array}{l}\text { Olive tree } \\
\text { Cultivar }\end{array}$ & Bioclimate & $\mathrm{N}$ & Area & Roundness & Circularity & Length & Width & $\begin{array}{l}\text { Length/ } \\
\text { Perimeter }\end{array}$ \\
\hline \multirow{2}{*}{ Chemlali } & Sub-humid & 10 & $0.51^{b}$ & $0.51^{b}$ & $.67^{\mathrm{a}}$ & $1.23^{\mathrm{a}}$ & $0.59^{b}$ & $2.1^{\mathrm{a}}$ \\
\hline & Semi-arid superior & 10 & $0.47^{\text {a }}$ & $0.45^{\mathrm{a}}$ & $.66^{\mathrm{a}}$ & $1.20^{\mathrm{a}}$ & $0.53^{a}$ & $2.3^{b}$ \\
\hline \multirow{2}{*}{ Besbessi } & Sub-humid & 20 & $1.02^{\mathrm{a}}$ & $0.54^{\mathrm{a}}$ & $0.64^{a}$ & $1.62^{b}$ & $0.85^{a}$ & $1.97^{b}$ \\
\hline & Semi-arid & 10 & $1.08^{a}$ & $0.71^{b}$ & $0.65^{a}$ & $1.42^{\mathrm{a}}$ & $0.99^{b}$ & $1.44^{\mathrm{a}}$ \\
\hline \multirow{2}{*}{ Chaïbi } & Sub-humid & 30 & $0.54^{\mathrm{b}}$ & $0.54^{\mathrm{a}}$ & $0.68^{a}$ & $1.20^{\mathrm{a}}$ & $0.62^{b}$ & $1.95^{\mathrm{a}}$ \\
\hline & Semi-arid & 10 & $0.47^{\mathrm{a}}$ & $0.53^{a}$ & $0.70^{a}$ & $1.13^{\mathrm{a}}$ & $0.57^{a}$ & $1.97^{\mathrm{a}}$ \\
\hline \multirow{2}{*}{ Chétoui } & Sub-humid & 30 & $0.67^{b}$ & $0.46^{\mathrm{a}}$ & $0.63^{a}$ & $1.46^{\mathrm{b}}$ & $0.64^{a}$ & $2.27^{b}$ \\
\hline & Semi-arid & 10 & $0.62^{a}$ & $0.49^{b}$ & $0.64^{a}$ & $1.36^{\mathrm{a}}$ & $0.64^{a}$ & $2.15^{\mathrm{a}}$ \\
\hline \multirow{2}{*}{ Gerboui } & Sub-humid & 40 & $0.68^{a}$ & $0.68^{a}$ & $0.71^{\mathrm{b}}$ & $1.17^{\mathrm{a}}$ & $0.78^{a}$ & $1.50^{\mathrm{b}}$ \\
\hline & Semi-arid & 10 & $0.74^{a}$ & $0.79^{b}$ & $0.68^{a}$ & $1.11^{\mathrm{a}}$ & $0.87^{b}$ & $1.28^{\mathrm{a}}$ \\
\hline \multirow{2}{*}{ Marsaline } & Sub-humid & 9 & $0.80^{\mathrm{b}}$ & $0.46^{\mathrm{a}}$ & $0.64^{a}$ & $1.56^{\mathrm{b}}$ & $0.69^{a}$ & $2.26^{\mathrm{b}}$ \\
\hline & Semi-arid & 10 & $0.66^{a}$ & $0.69^{b}$ & $0.70^{b}$ & $1.13^{\mathrm{a}}$ & $0.76^{b}$ & $1.49^{\mathrm{a}}$ \\
\hline
\end{tabular}


Table 7. Variation percentage and correlated variables with principal components (PC) on stone size and shape of wild and cultivated plants

\begin{tabular}{lcc}
\hline & PC 1 & PC 2 \\
\hline Variation percentage & 70.341 & 28.541 \\
Variables & & \\
Area & -0.282 & 0.012 \\
Perimeter & -0.084 & 0.0004 \\
Roundness & 0.145 & 0.216 \\
Circularity & 0.181 & 0.112 \\
Length & -0.056 & -0.058 \\
Width & -0.070 & 0.098 \\
length/width & 0.147 & -0.095 \\
Length & /perimeter & \\
\end{tabular}

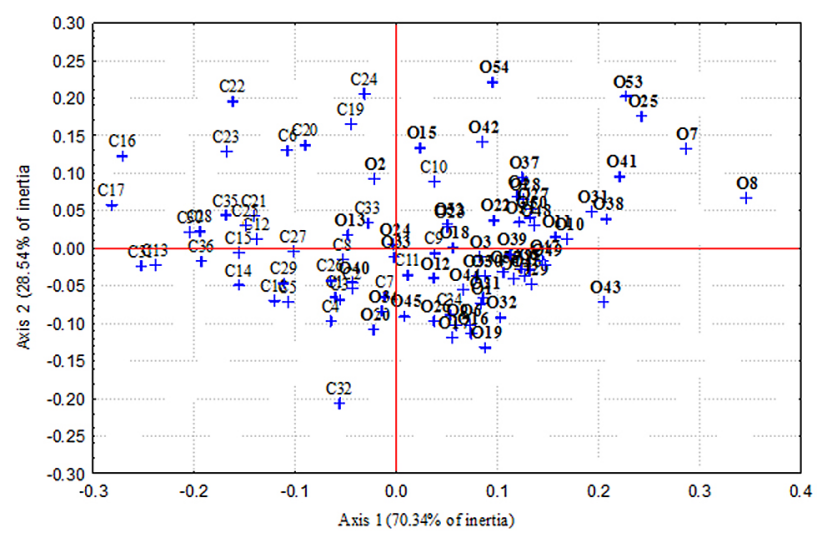

Fig. 4. Principal Component Analysis on stone size and shape of wild and cultivated olive trees, O: Oleaster (wild plants) and C: Cultivar (cultivated plants)

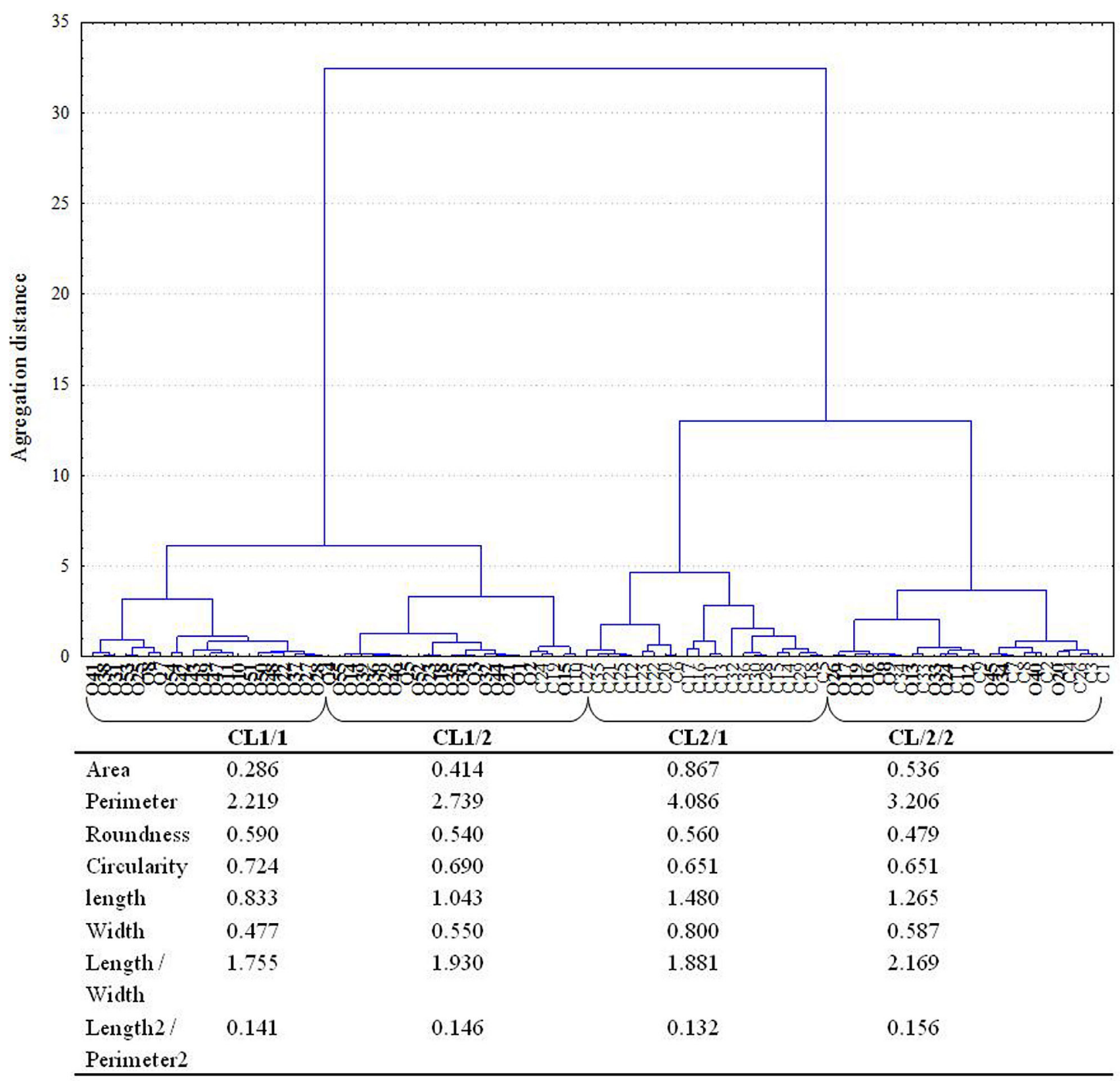

Fig. 5. Hierarchical analysis of wild and cultivated plants based on stone size and shape using the Euclidean distance and the Ward algorithm, O: Oleaster (wild plants) and C: Cultivar (cultivated plants) 
and C10 (Chaïbi). Sub-cluster CL2/1 contains only cultivated plants. The CL2/2 sub-cluster comprises wild (14 oleasters) and cultivated plants (10 cultivars) sharing closely some stone characters, and thus confirming the PCA plot.

The oleaster stones in CL1/1 were characterized by smaller area $\left(0.286 \mathrm{~cm}^{2}\right)$, perimeter $(2.21 \mathrm{~cm})$, length $(0.83 \mathrm{~cm})$ and width $(0.47 \mathrm{~cm})$ and were well separated from cultivars in CL2/1 characterized by higher area $\left(0.867 \mathrm{~cm}^{2}\right)$, perimeter $(4.08 \mathrm{~cm})$, length $(1.48 \mathrm{~cm})$ and width $(0.80 \mathrm{~cm})$.

The range of variation is much larger for seed size than shape. Figure 6 presents the range of variation in stone size for seeds of wild and cultivated plants. Figure 7 shows the range of variation of shape in stones of wild and cultivated plants.

\section{Discussion}

Quantification of seed shape requires the comparison of the seed image with a geometric figure (Cervantes et al., 2016). The method can be applied manually or in a semi-automated way based on automated image analysis programs. ImageJ program (Ferreira \& Wayne, 2010) allows to record automatically the values of circularity index and roundness in a high number of seed samples, thus being useful in the first approximation of seed shape quantification in a new species.

A semi-automated method for size and shape quantification in stone of Olea europaea, wild and cultivated, has been applied. As described before in the analysis of size and shape in Ricinus communis (Martín Gómez et al., 2016), the range of variation in size is much larger than for shape. Stones of the wild olive trees (oleaster) present a broad range of values and are smaller than the stones of the cultivated plants. Increased size in the cultivated varieties is expected due to: 1) Selection for higher size 2) Improved growth conditions in the cultivated varieties in comparison with the wild.

The PCA and cluster did not reveal a clear difference in shape between the two olive types wild and cultivated. Seed shape was not a target of selective pressures in during grapevine cultivation (Terral et al., 2010) and there is no shape difference between mature and immature stones (Terral et al., 2004).

The semi-automated method of image analysis gives higher values for circularity than for roundness but a large amount of variation is observed both for the wild and cultivated seeds with both magnitudes. Shape analysis may be done using different models for the wild and the cultivated stones, and it could be useful to apply particular geometric models to the groups obtained by cluster analysis.

Based on stone size and shape data segregate in two clusters that predominantly contain the wild and the cultivated olive trees (Figure 4,5 ) in a similar result as revealed by molecular markers (Hannachi et al., 2010; Belaj et al., 2011). An intermediate group comprised some oleasters and cultivars with closely related stones traits (CL1/2 and CL2/2). The two olive cultivars Gerboui (C19 and C24) were closely related with oleaster trees in CL1/2 independently to their bioclimatic location. The sub-cluster CL2/2 contains 8 oleasters and 14 olive cultivars. A similar

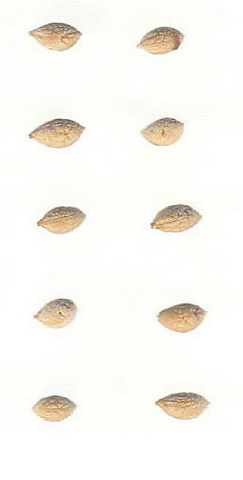

O08 - Ichkeul (Oleaster) Bioclimate.- Sub-humid

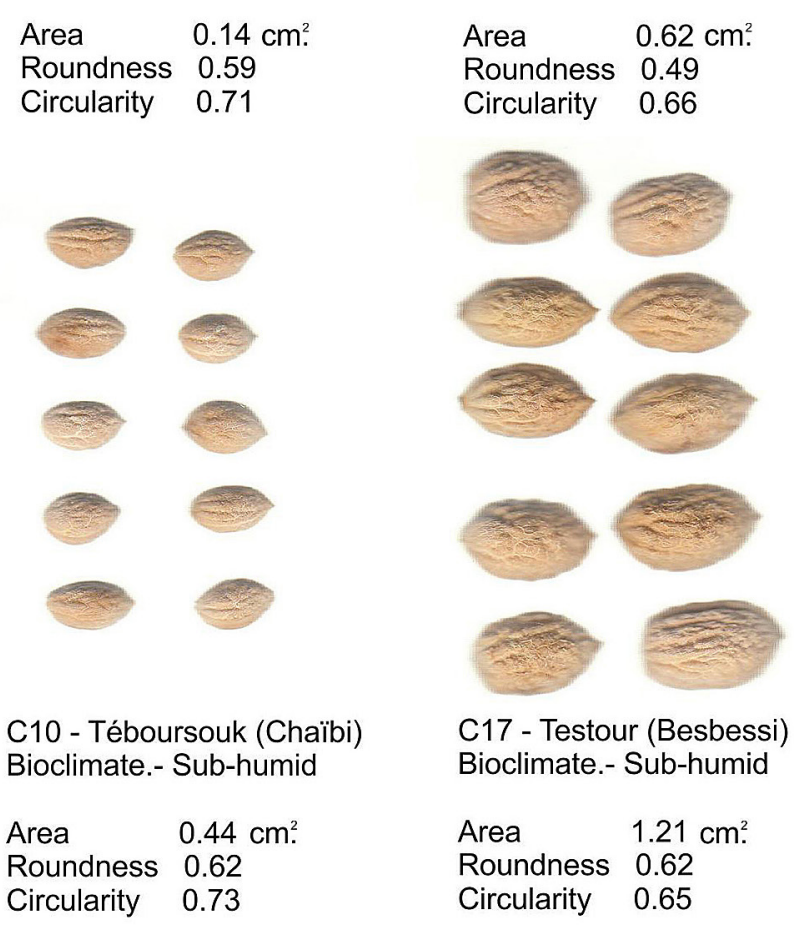

Fig. 6. Images of stones with minimum and maximum values of area

Top: Stones from wild populations $\mathrm{O} 08$ and O40. Seeds from $\mathrm{O} 08$ are the smaller and O40 larger among the wild populations.

Bottom: Stones from cultivated populations $\mathrm{C} 10$ and C17. Seeds from $\mathrm{C} 10$ are the smaller and C17 larger among the cultivated. 
continuous variation between cultivated and wild olive trees has been noted in studies based on morphological trait of tree, leaf, fruit and stone (Hannachi et al., 2008a, b) or based on molecular markers applied on Tunisian (Hannachi et al., 2010) and Spanish wild and cultivated olive trees (Belaj et al., 2010).

This close relationship may be explained by: i) the intermediate cultivars would be the results of local domestication. As indicated based on assignation and admixture analyses few of Tunisian cultivars are issued from the Tunisian oleaster (Breton et al., 2006a; Hannachi et al., 2008a). It is documented that several independent domestication events occurred in different regions of the Mediterranean basin (Terral et al., 2004; Terral \& Arnold-Simard, 1996); probably concomitantly with domestication in the Near East (Lanfranchi \& Bui, 1995; Terral, 2000). Therefore, the olive cultivars may display diversity according to domestication origins (Besnard et al., 2001; Breton et al., 2006b). ii) The phylogeography of the oleaster and cultivars trees is due to gene flow. The gene flow has continued between cultivated and wild
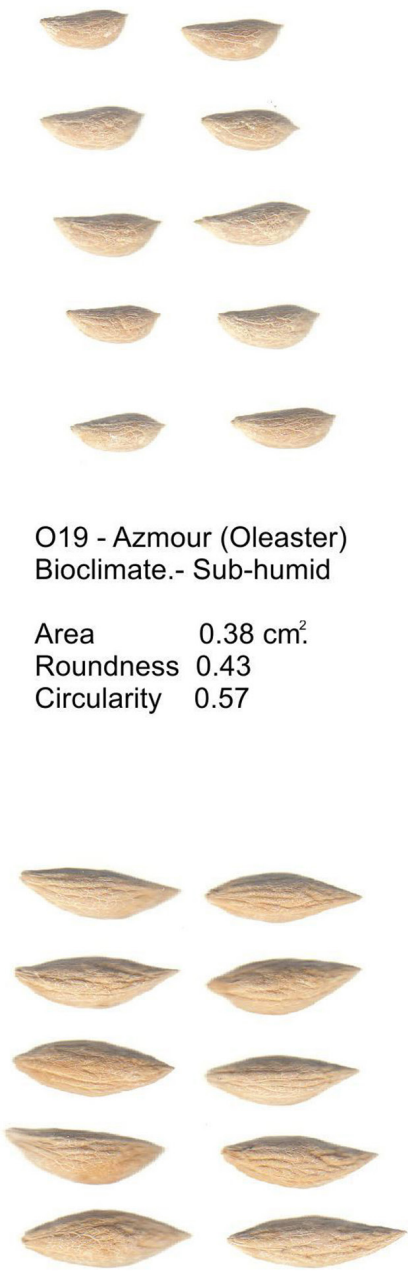

C32 - Testour (Neb Jmel) Bioclimate.- Sub-humid
Area
$0.72 \mathrm{~cm}^{2}$.
Roundness 0.34
Circularity 0.51

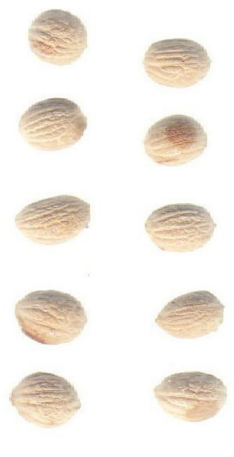

O54 - Laaroussa (Oleaster) Bioclimate.- Semi-arid

$\begin{array}{ll}\text { Area } & 0.34 \mathrm{~cm}^{2} . \\ \text { Roundness } & 0.75\end{array}$

Circularity $\quad 0.77$

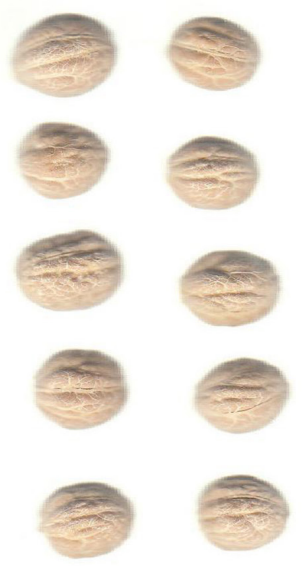

C22 - laaroussa (Gerboui) Bioclimate.- Semi-arid
Area
$0.74 \mathrm{~cm}^{2}$.
Roundness 0.79

Circularity 0.68

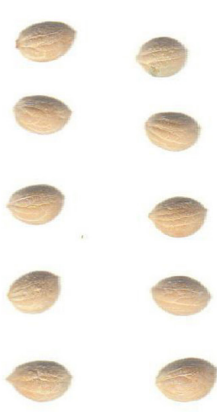

O53 - Tunis (Oleaster) Bioclimate.- Semi-arid -sup

Area $\quad 0.23 \mathrm{~cm}^{2}$.

Roundness 0.71

Circularity $\quad 0.80$

Fig. 7. Images of stones with minimum and maximum values of circularity and roundness

Top: Seeds from wild populations O19, O54 and O53. Seeds from O19have the lowest values of circularity and roundness; O54 and O53 the highest values of roundness and circularity respectively.

Bottom: Stones from cultivated populations C32, C22 and C24. Stones from C32 have the lowest values of circularity and roundness; $\mathrm{C} 22$ and $\mathrm{C} 24$ the highest values of roundness and circularity respectively. 
olives (Lumaret \& Ouazzani, 2001) and is locally detected between introduced and local oleasters and, in this case, they do not carry the same cytoplasm within oleasters (Breton et al., 2005); iii) the feral form of olive (Besnard et al., 2001; Mekuria et al., 2002; Breton et al., 2006a) escaping to cultivation practices and therefore losing some characters of stone. Feral olive forms were placed in intermediate position based on morphological trait of tree, leaf, fruit and stone (Hannachi et al., 2010). Genetically, feral olives can be shown to be progeny of nearby cultivated olive trees (Mekuria et al., 2002). Some endocarp criteria such as perimeter and area with others were found to be useful for taxonomic analysis applied of Prunus (Depypere et al., 2007).

In the wild populations, an increase in size is observed in some trees grown in the arid region. This is unexpected and may be due either to particular micro-environmental climatic factors concerning these trees or it may be related to the fact that these stones have also reduced values of circularity and roundness. Thus, larger sizes may be associated/ or required with elongated seeds, and this later character may be useful in the arid conditions, for example helping to seed transport or survival under occasional flows or in response to water. Differences in size and shape were observed among localities in the four climatic regions.

In general there are climatic and geographic factors that determine changes in seed size and shape. For example a trend has been observed in seeds of the same species to decrease in size with latitude (Soper Gorden et al., 2016). However, it has been also noted the genetic primarily additive effect on seed size and shape; indeed a minimum of three or four genetic factors or blocks of genes appears to control seed heritability of seed size of Sorghum vulgare of the order of $60 \%$ (Voigt et al., 1966).

Also, seeds of plants grown in the desert have been reported to be smaller in some instances. For example, a relationship between seed size and soil persistence has been described such that persistent seeds are smaller (Peco et al., 2003). The accurate quantification of seed diversity is important from an ecological perspective. Seed size is embedded of attributes defining the life history of a plant species (Westoby, 1998). According to the geographical localities, the wild olive populations are found in a high diversity of environments with different altitudes and soils and were reported to contain more variability than the cultivated one (Belaj et al., 2010).

\section{References}

Baccouri B, Guerfel M, Zarrouk W, Taamalli W, Daoud D \& Zarrouk M (2011) Wild olive (Olea europaea
L.) selection for quality oil production. Journal of Food Biochemistry 35: 161-176.

Bartolini G, Prevost G, Messeri C \& Carignani G (1998) Olive germplasm cultivars and world-wide collections. FAO Seed \& Plant Genetic Resources Service. Plant production and Protection division, Roma.

Belaj A, Munoz-Diez C, Baldoni L, Porceddu A, Barranco D \& Satovic Z (2007) Genetic diversity and population structure of wild olives from North-Western Mediterranean assessed by SSR markers. Annals of Botany 100: 449-458.

Belaj A, Muňoz-Diez C, Baldoni L, Satovic Z \& Barranco D (2010) Genetic diversity and relationships of wild and cultivated olives at regional level in Spain. Scienctia Horticulturae 124: 323-330.

Belaj A, León L, Satovic Z \& De la Rosa R (2011) Variability of wild olives (Olea europaea subsp. europaea var. sylvestris) analyzed by agro-morphological traits and SSR markers. Scienctia Horticulturae 129: 561-569.

Besnard G, Baradt P \& Bervillé A (2001) Genetic relationships in the olive (Olea europaea L.) reflect multilocal selection of cultivars. Theoretical and Applied Genetics 102: 251-258.

Besnard G, Khadari B, Villemur P \& Bervillé A (2000) Cytoplasmic male sterility in the olive (Olea europaea L.). Theoretical and Applied Genetics 100: 1018-1024.

Breton C, Médail F, Pinatel C \& Bervillé A (2005) Olive-oleaster gene flow and risks of ferality in olive, chapter 15: Crop ferality and volunteerism, a threat to food security in the transgenic era? (ed. by J Gressel) CRC Press, Boca Raton, USA, pp. 231-234.

Breton C, Tersac M \& Bervillé A (2006a) SSR genetic diversity in wild olive (oleaster, Olea europaea L.) suggests several Plio-Pleistocene refuge zones in the Mediterranean Basin and gene flow with olive. Journal of Biogeography 33: 1916-1928.

Breton C, Besnard G \& Bervillé A (2006b) Using multiple types of molecular markers to understand olive phylogeography: Documenting domestication, new genetic and archaeological paradigms (ed. by MA Zeder, D Decker-Walters, D Bradley \& B Smith) University of California Press, pp. 143-152.

Camps-Fabrer H (1997) La culture de l'olivier en Afrique du Nord. Evolution et histoire: Encyclopédie Mondial de l'Olivier, Madrid, Espagne, pp. 30-33.

Cervantes E, Martín JJ, Ardanuy R, de Diego JG \& Tocino Á (2010) Modeling the Arabidopsis seed shape by a cardioid: efficacy of the adjustment with a scale change with factor equal to the Golden Ratio and analysis of seed shape in ethylene mutants. Journal of Plant Physiology 167: 408410. 
Cervantes E, Martín JJ, Chan PK, Gresshoff PM \& Tocino Á (2012) Seed shape in model legumes: approximation by a cardioid reveals differences in ethylene insensitive mutants of Lotus japonicus and Medicago truncatula. Journal of Plant Physiology 169: 1359-1365.

Cervantes E, Martín JJ \& Saadaoui E (2016) Updated methods for seed shape analysis. Scientifica: ID5691825, 10 pages http://dx.doi. org/10.1155/2016/5691825.

Costa C (1998) Olive production in South Africa: A handbook for olive growers. Agricultural Research Council Press, Pretoria.

Cox EP (1927) A method of assigning numerical and percentage values to the degree of roundness of sand grains. Journal of Paleontology 1: 179-183.

Dabbou S, Dabbou S, Selvaggini R, Urbani S, Taticchi A, Servili M \& Hammami M (2011) Comparison of the chemical composition and the organoleptic profile of virgin olive oil from two wild and two cultivated Tunisian Olea europaea. Chemistry \& Biodiversity 8: 189-202.

Depypere L, Chaerle P, Mijnsbrugge KV \& Goetghebeur P (2007) Stony endocarp dimension and shape variation in Prunus section Prunus. Annals of Botany 100: 1585-1597.

Erre P, Chessa I, Mŭnoz-Diez C, Belaj A, Rallo L \& Trujillo I (2010) Genetic diversity and relationships between wild and cultivated olives (Olea europaea L.) in Sardinia as assessed by SSR markers. Genetic Resources and Crop Evolution 57: 41-54.

Ferreira T \& Wayne R (2010) Imagej user guide ver-

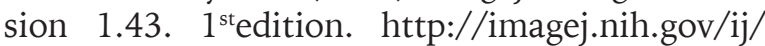
docs/guide/index.html\#.

Green PS (2002) A revision of Olea L. (Oleaceae). Kew Bulletin 57: 91-140.

Hannachi H, Breton C, Msallem M, Ben El Hadj S, El Gazzah M \& Bervillé A (2008a) Differences between native and introduced olive cultivars as revealed by morphology of drupes, oil composition and SSR polymorphisms: A case study in Tunisia. Scienctia Horticulturae 116: 280-290.

Hannachi H, Breton C, Msallem M, Ben El Hadj S, El Gazzah M \& Bervillé A (2008b) Are olive cultivars distinguishable from oleaster trees based on morphology of drupes and pits, oil composition and microsatellite polymorphisms? Acta Botanica Gallica 155: 531-545.

Hannachi H, Breton C, Msallem M, Ben El Hadj S, El Gazzah M \& Bervillé A (2010) Genetic relationships between cultivated and wild olive trees (Olea europaea L. var. europaea and var. sylvestris) based on nuclear and chloroplast SSR markers. Natural Resources 1: 95-103.

Hannachi H, Nizar N, Walid E, Nizar T, Ali F \& Monji M (2013) Fatty acids, sterols, polyphenols and chlorophylls of olive oils obtained from Tunisian wild olive trees (Olea europaea L. var. sylvestris). International Journal of Food Properties 16: 12711283.

Hannachi H \& Marzouk S (2012) Flowering in the wild olive (Olea europaea L.) tree (oleaster): phenology, flower abnormalities and fruit set traits for breeding the olive. African Journal of Biotechnology 11: 8142-8148.

Idrissi A \& Ouazzani N (2003) Apport des descripteurs morphologiques à l'inventaire et a l'identification des variétés d'olivier (Olea europaea L.). PGR Newsletter 136: 1-10.

Krichene D, Taamalli W, Daoud D, Salvador MD, Fregapane G \& Zarrouk M (2007) Phenolic compounds, tocopherols and other minor components in virgin olive oils of some Tunisian varieties. Journal of Food Biochemistry 31: 179-194.

Lanfranchi F \& Bui TM (1995) Oléastre et lentisque de Corse et de Sardaigne, deux plantes oléagineuses sauvages dans l'économie Néolithique: Olio Sacro e profano, tradizione olearie in Sardinia e Corsica, M. Atzori and A. Vodret, Editrice Democratica Sarda. Sassari, Italy.

Lumaret R \& Ouazzani N (2001) Plant genetics: Ancient wild olives in Mediterranean forests. Nature 413: 700 .

Martin Gómez JJM, Saadaoui E \& Cervantes E (2016) Seed shape of castor bean (Ricinus communis L.) grown indifferent regions of Tunisia. Journal of Agriculture and Ecology Research International 8: 1-11.

Martín JJ, Tocino A, Ardanuy R, de Diego JG \& Cervantes E (2014) Dynamic analysis of Arabidopsis seed shape reveals differences in cellulose mutants. Acta Physiologiae Plantarum 36: 15851592.

Mekuria GT, Collins G \& Sedgley M (2002) Genetic diversity within an isolated olive (Olea europaea L.) population in relation to feral spread. Scientia Horticulturae 94: 91-105.

Noormohammadi Z, Trujillo I, Belaj A, Ataeid S \& Hosseini-Mazinand M (2014) Genetic structure of Iranian olive cultivars and their relationship with Mediterranean's cultivars revealed by SSR markers. Scientia Horticulturae 178: 175-183.

Peco B, Traba J, Levassor C, Sánchez AM \& Azcárate FM (2003) Seed size, shape and persistence in dry Mediterranean grass and scrublands. Seed Science Research 13: 87-95.

Riley NA (1941) Projection sphericity. Journal of Sedimentary Petrology 11: 94-97.

Rotondi A, Magli M, Ricciolini C \& Baldoni L (2003) Morphological and molecular analyses for the characterization of a group of Italian olive cultivars. Euphytica 132: 129-137.

Rovner I \& Gyulai F (2007) Computer-assisted morphometry: a new method for assessing and dis- 
tinguishing morphological variation in wild and domestic seed populations. Economic Botany 61: 154-172.

Saadaoui E, Gomez MJJ \& Cervantes E (2013) Intraspecific variability of seed morphology in Capparis spinosa L. Acta Biologica Cracoviensia Series Botanica 55: 99-106.

Saadaoui E, Martín JJ, Bouazizi R, Ben Romdhane C, Grira M, Abdelkabir S, Khouja ML \& Cervantes Ruiz de la Torre E (2015) Phenotypic variability and seed yield of Jatropha curcas L. introduced to Tunisia. Acta Botanica Mexicana 110: 119-134.

Schwartz H (1980) Two-dimensional feature-shape indexes. Mikroskopie 37(Suppl): 64-67.

Soper Gorden NL, Winkler KJ, Jahnke MR, Marshall E, Horky J, Huddelson C \& Etterson JR (2016) Geographic patterns of seed mass are associated with climate factors, but relationships vary between species. American Journal of Botany 103: 60-72.

Terral JF (2000) Exploitation and management of the olive tree during Prehistoric times in Mediterranean France and Spain. Journal of Archaeological Science 27: 127-133.
Terral JF, Alonso N, Buxo R, Capdevila RB, Chatti N, Fabre L, Fiorentino G, Marinval P, Perez Jorda G, Pradat B, Rovira N \& Alibert P (2004) Historical biogeography of olive domestication (Olea europaea L.) as revealed by geometrical morphometry applied to biological and archaeological material. Journal of Biogeography 31: 63-77.

Terral JF \& Arnold-Simard G (1996) Beginnings of olive cultivation in eastern Spain in relation to Holocene bioclimatic changes. Quaternary Research 46: 176-185.

Terral JF, Tabard E, Bouby L, Ivorra S, Pastor T, Figueiral I, Picq S, Chevance JB, Jung C, Fabre L, Tardy C, Compan M, Bacilieri R, Lacombe T \& Patrice T (2010) Evolution and history of grapevine (Vitis vinifera) under domestication: new morphometric perspectives to understand seed domestication syndrome and reveal origins of ancient European cultivars. Annals of Botany 105: 443-455.

Voigt RL, Gardner CO \& Webster OJ (1966) Inheritance of seed size in Sorghum, Sorghum vulgare Pers. Crop Science 6: 582-86.

Westoby M (1998) A leaf-height-seed (LHS) plant ecology scheme. Plant and Soil 199: 213-227. 